Research Article

\title{
Design and Performance Simulation of Computer Control System for Automatic Monitoring of Upper Computer Communication Operation State
}

\author{
Li Yang $\mathbb{D}^{1}$ and Huitao Zhang $\mathbb{D}^{2}$ \\ ${ }^{1}$ School of Electrical and Mechanical, Shijiazhuang University, Hebei, Shijiazhuang 050035, China \\ ${ }^{2}$ School of Computer Science, Shijiazhuang University, Hebei, Shijiazhuang 050035, China \\ Correspondence should be addressed to Huitao Zhang; 12116317@bjtu.edu.cn
}

Received 22 October 2021; Revised 8 November 2021; Accepted 10 November 2021; Published 6 December 2021

Academic Editor: Shan Zhong

Copyright (C) 2021 Li Yang and Huitao Zhang. This is an open access article distributed under the Creative Commons Attribution License, which permits unrestricted use, distribution, and reproduction in any medium, provided the original work is properly cited.

\begin{abstract}
The upper computer communication operation state automatic monitoring system is mainly used to remotely monitor the equipment, obtain various parameter indexes in the operation process of remote equipment, realize remote monitoring and fault diagnosis, and improve the management efficiency of decentralized equipment. This paper completes the design of communication, data storage, query, and other subsystems of upper and lower computers. The lower computer establishes a data channel with the OPC server through the MPI protocol and uploads the collected data to the OPC server in real time. The upper computer reads the data through the OPC server and displays the changes of monitored parameters in real time through the monitoring interface, so as to give an alarm under abnormal conditions. In addition, since the default database of Kingview is access, considering that the Microsoft Access database can store up to $2 \mathrm{G}$ of content, in order to upgrade and expand the subsequent system, SQL Server database is selected for data query, backup, and saving. The parameter setting method of communication control system is analyzed, the simulation model of industrial boiler control system is established by using Matlab/Simulink, and the interface between host computer software (IBCCS-e) and the model is provided. This paper analyzes the results of communication parameter adjustment. The simulation results show that the industrial boiler computer control system (IBCCS) has stable performance, low cost, convenient operation, and good maintainability. After further improvement, it has certain application value in the operation transformation of new small- and medium-sized boilers and original boilers.
\end{abstract}

\section{Introduction}

With the continuous expansion of the operating scale of industrial enterprises, only realizing the control of various field devices can no longer meet higher requirements, and the real-time response to the working conditions of the field devices has more practical significance [1]. As the company's on-site environment is becoming more and more complex, and the targets to be monitored are more diverse, the structure of the monitoring system becomes more and more decentralized. The traditional programmable logic device to directly control field devices can no longer meet the decentralized requirements of the system topology [2]. However, in the application that combines PLC and fieldbus technology after development, only PLC programming is used to realize the control of the device, so industrial monitoring systems began to tend to use computer monitoring systems [3].

The computer remote monitoring system is a monitoring network system that combines multimedia technology, network technology, and industrial automation technology with a computer as the core [4]. Its obvious advantage lies in remote monitoring and effectiveness. The remote monitoring system can obtain on-site information and send it to the monitoring center and make it reach the personal desktop PC through the computer network, so that it can be easily integrated with the information management system to better serve management and improve management level 
and efficiency. The use of computers to monitor and control industrial production processes is widely used in modern society and plays an extremely important role in the safety and economy of industrial production operations. The production situation on site can be reproduced in time in front of decision-makers and managers away from the site, and the real-time data on site can be provided to functional departments at all levels to form charts to integrate production control and modern management [5]. The computer monitoring system can easily turn the scattered, large-area console-type monitoring into a centralized screen monitoring, which greatly reduces the work intensity of the operators [6].

This paper studies the overall architecture of the host computer communication operation state automatic monitoring system, provides a reasonable solution according to the specific situation of this paper, analyzes the network topology and architecture of the wireless monitoring system, determines the selection of measurement parameters, and identifies the radio wave propagation characteristics and signal maintenance methods of the communication system. The host computer system of PLC monitoring system of node boiler, the remote monitoring system of centralized control center, and the networking mode of 19 monitoring nodes are designed. The storage, calling, and permission setting of boiler operation state parameters are clarified. The remote monitoring is described. The working method of PID parameter setting module in host computer communication software (IBCCS-e) is introduced in detail. Then, the simulation model of industrial boiler computer control system is established by using Matlab/Simulink, and the data interface between Delphi and the simulation model is realized. Based on the simulation model, the parameter setting simulation experiments of water supply communication control system and combustion control system are carried out, and the experimental results are analyzed.

\section{Related Work}

In the centralized computer monitoring structure, the host computer in the central control room processes through the host computer configuration software system the various analog signals and switch signals that are introduced into the central control room host interface via the relevant cables, and then the form is issued to realize the background related data processing and real-time control of on-site equipment [7]. The monitoring system uses a single host; once the host fails, the entire system will be paralyzed; all signals are processed by a CPU, and the real-time performance is difficult to guarantee. Related scholars pointed out that, for systems with scattered signal collection points such as small hydropower stations, a large amount of cables must be used for signal transmission, which increases the cost of the entire system design; the existence of electromagnetic interference seriously affects the reliability and measurement of the system [8].

There are relatively few researches on the real-time database and cloud platform of APF operation and management, and the structure analysis is not clear. In the design of database-related strategies, existing or backward strategies are mostly used without reasonable analysis [9]. The data of the APF running real-time monitoring system has its own characteristics, but the existing real-time database and relational database are not specific to the data processing generated by the APF operation. The real-time database and the relational database are part of the APF running real-time monitoring platform, and the APF operation real-time monitoring platform is consistent to be integrated into the APF operation real-time monitoring platform [10]. Therefore, traditional industrial real-time databases and relational databases are not suitable for real-time data processing of APF running real-time monitoring systems. In terms of APF equipment monitoring, power electronic technology and cloud computing technology are widely used to realize realtime data collection and intelligent processing [11]. The APF monitoring system integrates database technology and computer technology, effectively stores and manages data, and provides the system with corresponding basic data for secondary calculation and processing, thereby improving the automation of the APF real-time monitoring system.

With the gradual expansion of the network scale, the demand for network monitoring models is gradually changing [12]. Currently, commonly used network monitoring models are centralized network monitoring model based on $\mathrm{C} / \mathrm{S}$, network monitoring model based on Web mode, network monitoring model based on distributed mode, and network monitoring model based on multiagent mode [13]. The network monitoring model has gradually changed from a traditional centralized model to a distributed model, and from a single model to a hybrid model.

At present, domestic and foreign monitoring software can be divided into three parts. The first part is foreign professional monitoring software represented by HP and IBM. Its management products focus on the server monitoring of their own products, mainly through agent monitoring. The performance of the monitoring software is relatively stable. The second part is the domestic companies represented by MXsoft, Youlong, and North Tower. These companies rely on secondary development and localized technical support and services to obtain more and more applications, and their product technologies are gradually becoming more popular. The third part is composed of other related software vendors. The network monitoring software analyzes from a technical point of view and realizes the comprehensive management mode from the product's own tool management to the efficient integration, and distributed combination is the future development trend of information system management technology $[14,15]$. These commercial monitoring software tools are easy to operate and have stable performance, but they also have shortcomings such as high closure, strong pertinence, poor scalability, and high cost.

At present, the domestic condition monitoring system is based on a microcomputer and centered on software development $[16,17]$. It has multiple functions, great flexibility, wide application, and low system price, which can better complete the purpose of condition monitoring of hydropower units. However, there are still some problems such as weak hardware functions, mainly roving monitoring, 
and poor real-time performance. Field wiring workload is large, signal anti-interference issues need to be carefully considered, it is difficult to adapt to the harsh environment of the industrial site, and because each manufacturer uses different technologies, it is difficult to achieve interchange and interoperability with each other, and compatibility is not good [18]. How to solve these problems and further improve and perfect the performance of the condition monitoring system has become an urgent problem for system designers $[19,20]$.

\section{The Overall Architecture of the Automatic Monitoring System for the Running Status of the Upper Computer}

3.1. The Network Topology of the Host Computer Wireless Monitoring System. This paper proposes a remote monitoring system for equipment based on wireless communication. Its network topology is shown in Figure 1. It can be seen from the figure that the overall structure of the monitoring system is mainly composed of three parts, including information collection and transmission equipment, data storage and analysis equipment, and remote automatic monitoring system software.

The monitoring terminal collects and analyzes the information during the operation of the equipment in real time and periodically transmits the collected data wirelessly to the server; the data storage and analysis equipment stores and analyzes the data in the server. Data display and remote automatic monitoring system software processing analyses are convenient for managers to carry out real-time monitoring and fault trend prediction; at the same time, the data in the server can also be published to various remote terminals (such as mobile phones and computers) through the Internet. With software, supervisors can obtain real-time monitoring equipment operating behavior data information from all over the world, which will greatly benefit the future integration with smart home systems and realize the ubiquitous "Internet of Things" communication era [21].

\subsection{Host Computer Wireless Monitoring System Architecture.} From the system architecture of the monitoring system, it can be divided into the bottom field data collection layer, the wireless data transmission layer, and the top application service layer, as shown in Figure 2.

The data acquisition layer relies on various sensors for real-time acquisition and conversion of data from various measuring points of field equipment. The data transmission layer is composed of wireless terminals and concentrators, without on-site wiring and has high flexibility and scalability. The concentrator and the upper computer are relatively fixed. The upper computer is generally located in the monitoring room, and the concentrator is located above the monitoring room to receive data. The concentrator directly transmits on-site information to the upper computer for data storage and processing through a cable connection. The application service layer can realize the database management of the monitoring system and respond to client requests. It can provide authorized data services to corporate intranet users via the local area network and can provide corresponding data services to authorized personnel in various places via the Internet [22].

3.3. Analysis of System Radio Wave Propagation Characteristics. The signal received by the concentrator is composed of direct waves, ground reflection waves, and obstacle diffraction waves. The height of the concentrator is $\mathrm{hb}$, and the height of the wireless transmitter is $\mathrm{hm}$. The propagation distances of the radio waves are $d, d_{1}$, and $d_{2}$, respectively. Then, the signal field strength $E_{r}$ at the receiving point of the concentrator is the combination of the above three waves:

$$
E_{r}=E_{0} \cdot\left|1-e^{-2 \pi j / \lambda} \cdot \Delta d_{1}-e^{-2 \pi j / \lambda} \cdot \Delta d_{2}\right| .
$$

Among them, $\Delta d_{1}=d_{1}-d, \Delta d_{2}=d_{2}-d, E_{0}$ is the direct wave field strength, $\lambda$ is the working wavelength, $a_{1}$ is the attenuation coefficient of the ground reflected wave relative to the direct wave, and $a_{2}$ is the obstacle diffraction wave relative to the direct wave.

In on-site communication, the propagation of radio waves will encounter various reflections, scattering, and absorption. The received signal is not only composed of direct waves, but a combination of multiple radio waves, and the received point field strength is an interference field. Suppose that the carrier signal transmitted by the wireless transmitter is

$$
S_{0}(t)=a_{0} \cdot \lambda \cdot e^{j \phi_{0}-w_{0} t} .
$$

After reflection, scattering, and absorption, the carrier signal of the electric wave reaching the receiving point is

$$
S(t)=\prod_{i=0}^{n-1} \Phi_{i} \cdot S_{i}(t) .
$$

Among them, $S_{i}(t)$ is the $i$-th carrier signal arriving at the receiving point, $a_{i}$ is the amplitude of the $i$-th signal, and $\Phi_{i}$ is the phase change value of the $i$-th signal. Therefore,

$$
S(t)=\prod_{i=0}^{n-1} a_{i} \cdot e^{\phi_{i}-k v t \sin \theta_{i}+k w_{0} t} .
$$

Among them, $k$ is the phase shift constant, $k=2 \pi / \lambda ; \nu$ is the moving speed of the device; $\theta_{i}$ is the incident angle of the radio wave; $k \nu \cos \theta_{i}$ is the angular frequency shift caused by the Doppler effect.

Since there are many factors that cause reflection in the environment, $S(t)$ is a combination of multiple radio waves. In the above formula, $n$ is infinite, so it can be rewritten as the following formula:

$$
S(t)=A_{t} j S \cdot e^{j \Psi_{t}} \cdot e^{j \omega_{0} t-\Psi_{i}} \cdot
$$

Among them, 

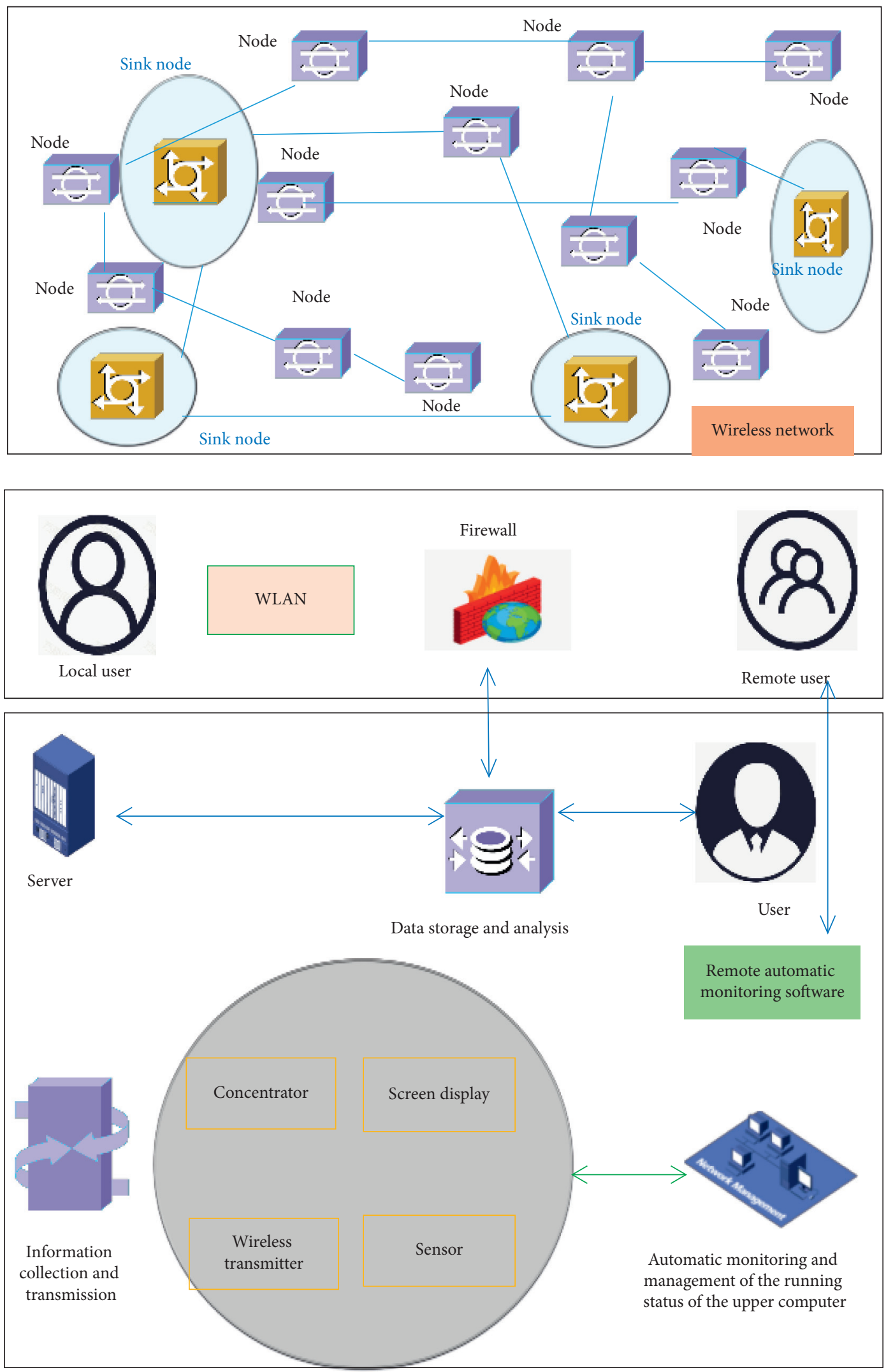

FIgURE 1: Wireless monitoring system network architecture diagram. 

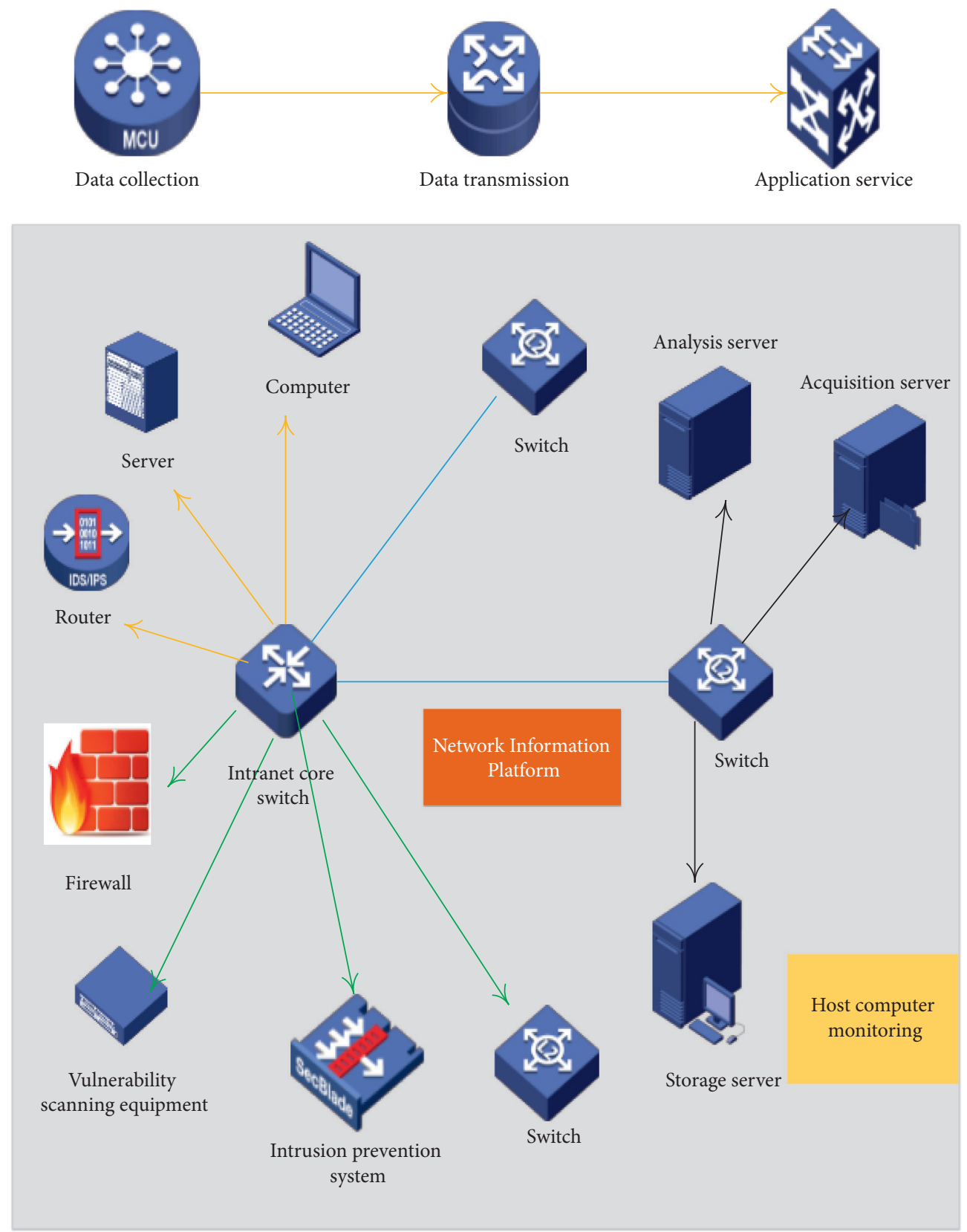

Figure 2: Wireless monitoring system architecture.

$$
\begin{aligned}
A_{t} & =\prod_{i=0}^{n-1} a_{i} \cdot \sin \Psi_{i}, \\
S & =\prod_{i=0}^{n-1} a_{i} \cdot \tan \Psi_{i}, \\
\Psi_{i} & =k v t \sin \theta_{i}-\phi_{i}, \\
\Psi_{t} & =\arccos \left(\frac{S}{R}\right) .
\end{aligned}
$$

Among them, $R$ and $S$ are independent variables composed of the sum of two infinitely large numbers of random variables, which obey a normal distribution, respectively. Their composite wave (that is, the amplitude $A_{t}$ of the carrier signal at the receiving point) obeys the Rayleigh distribution, and the phase $\Psi_{t}$ obeys the uniform distribution. In the field environment, if the monitoring point on the device is constantly moving, then as the monitoring point moves, the relative position between the wireless transmitter and the obstacle will continue to change, making the amplitude of the carrier signal received by the concentrator, causing the fading of the propagating signal. 


\section{The Design of the Host Computer Monitoring System}

4.1. Monitoring System Design Scheme. In view of the actual monitoring requirements of the hot water boiler of the group company, each node boiler is monitored by a host computer during the system design, and its monitoring system is also developed by Kingview software. On this basis, the main control computers of all nodes are networked, and the information is integrated and displayed in real time on the screen of the group company's hot water boiler operation status monitoring center. It should be pointed out that the system development of the monitoring center is also completed in the Kingview environment. This section describes the design and description of the development of the host computer monitoring system for a certain node's boiler operating status [23].

The design goal of the host computer monitoring system is to dynamically display the changes of important operating parameters of the boiler in the monitoring screen in real-time. You use Kingview's rich library and library development tools to monitor the operating status of a group company's boiler and create it according to the actual needs. The new screen is configured using the library, and then the corresponding relationship between the screen object and the system variable parameters is established through the animation connection and the command language. In addition, the system functions such as the control and command language provided by the Kingview are used in the boiler running state, the data is stored, and data reports are generated. Since Kingview is a real clientserver model supported by the network as a platform, the monitoring node can be designed as an I/O server according to the network size. In the design of the boiler operating status monitoring system of a group company, using this feature of Kingview, the host computer is used as a client. The status parameters collected by the PLC of each node can realize realtime monitoring of the operation of all hot water boilers.

The host computer monitoring system adopts a modular design concept, which is mainly composed of a login interface, a main monitoring interface, a real-time trend curve interface, a real-time report interface, and an alarm window interface. The structure diagram is shown in Figure 3. In the main interface management system, the real-time data of the main parameters can be inquired in real time.

We select the configuration menu in the Kingview's project browser and set according to the following process: configuration $\longrightarrow$ user configuration $\longrightarrow$ user and security configuration. Three security levels have been set up: system administrators, management staff, and boiler workers. The security zone of the system administrator is configured as A, which is the highest level, followed by management staff and boiler workers. We set different system login passwords for personnel with three security levels. For example, when turning on the boiler control system, you can select the corresponding user type through the mouse or directly click on the touch screen according to the type of user.

You can also modify the password on the boot interface. You click the "Modify Password" button to pop up a

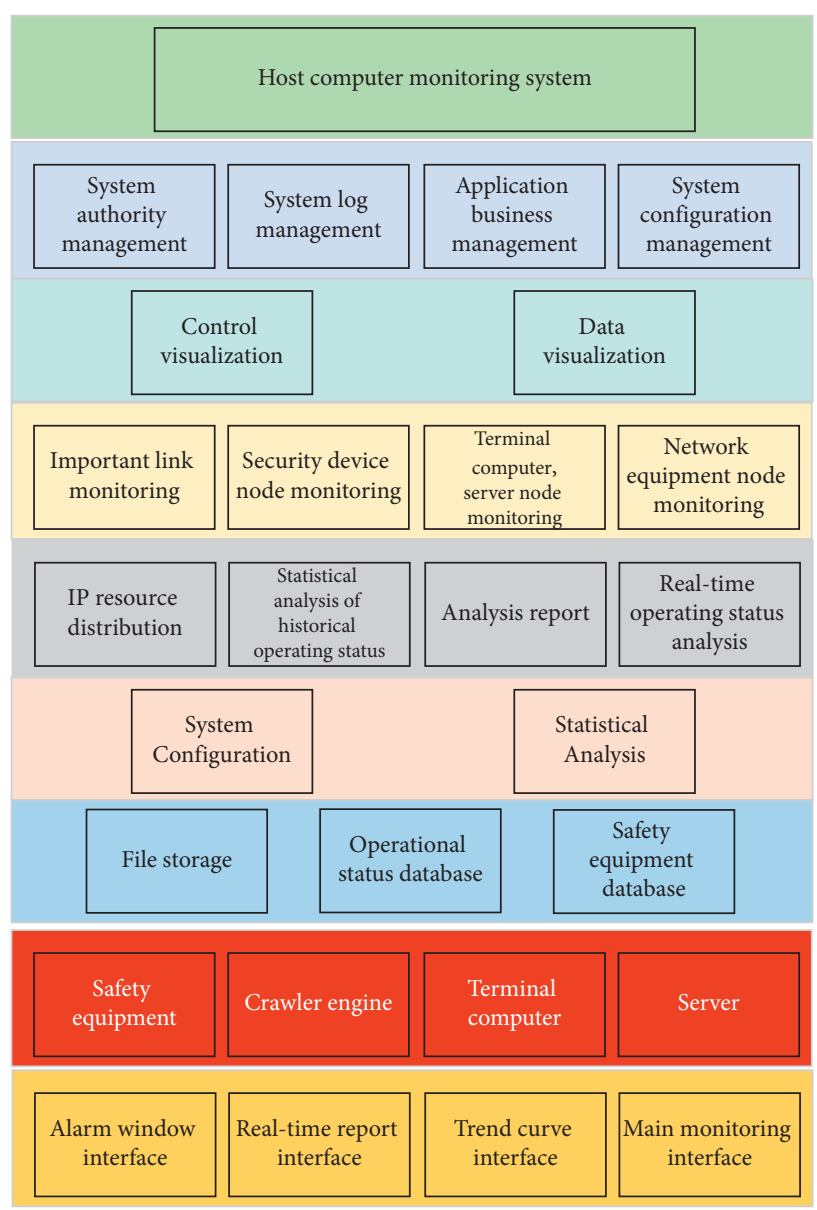

FIgURE 3: The structure of the host computer monitoring system.

subinterface to implement the password modification function. After entering the password on the boot interface, you press the "Enter" key on the keyboard. If the password is correct, the system enters the boiler system control interface. If the password is incorrect, a window will pop up to prompt that the password is incorrect, and the input can be continued after confirmation operation. If it is incorrect three times, it will take half an hour to reenter the password to be effective.

4.2. Monitoring and Alarm Design. In the operation of the monitoring system, if there is an alarm, the alarm light turns red. After the staff finds an alarm event, they can use the alarm elimination button to eliminate the alarm and perform inspection and troubleshooting. The water inlet and outlet pipes can dynamically display the direction of water flow. At the same time, the user can directly enter the window screen by clicking each window button to view the current data display status. The host computer establishes communication with the PLC through Kingview and uploads the data collected by the PLC to the Kingview database through OPCServer in real time. The upper computer displays the collected data in real time. The alarm screen records the event date, event time, alarm date, alarm time, 
alarm variable name, alarm type, alarm value/old value, and restored value/new value through the alarm window; the real-time trend screen is used to display the current working parameters of the hot water boiler. The historical data record of each monitoring equipment parameter can be called up in the historical curve screen and displayed in the form of a curve, so that the staff can check the historical data record at any time at any time; the report screen can query and export historical reports and real-time reports.

On one hand, the function of the alarm display is to drive the signal light for the duty personnel to check; on the other hand, the alarm is recorded in the data table for the administrator and supervisor to check. The alarm confirmation button is mainly to check whether the staff on duty found and dealt with the alarm. It is used to distinguish responsible accidents. The alarm system is convenient, flexible, reliable, and easy to expand. Distributed alarm management provides multiple management functions. Alarms and events have multiple output methods: files, databases, printers, and alarm event alarms including switch alarms and analog alarms. According to the data types collected by the field equipment, there are two types of alarms: one is a digital alarm; the other is an analog alarm. Through the communication channel uploaded to the alarm window of the monitoring host, the user can intuitively monitor and view the alarm information of the system and operate and control the running status of each subsystem. You select a button box in the KingView toolbar, determine the size, then select the animation connection realized by clicking the button, and select the required function in the animation connection.

When an alarm is generated, the alarm window automatically pops up on the desktop, and the report of the alarm information also pops up on the desktop. The alarm value exceeding the upper and lower limits can be seen in the alarm information report, and the sound, light, and voice alarms are automatically activated. The upper computer system will record the alarm time, and the lower computer will transmit the alarm record to the upper computer database in real time and display it in the form of a report. When the number of records in the buffer reaches the upper limit preset by the system, the system will automatically transfer the alarm information to the alarm database, and the printer can print the alarm records in the form of reports in real time.

4.3. Monitoring Data Storage and Query Design. Based on the overall architecture of a hot water boiler monitoring system of a group company, this section gives a further explanation of the database design. Because this monitoring system adopts a distributed structure, at each monitoring node, the real-time operating parameters of the corresponding boiler are first stored in the database of the host computer of the PLC monitoring subsystem of the node. This feature provides data acquisition for the monitoring center of the group company. There are two ways. One is that the real-time data obtained from the sensor system is stored in the node database and displayed on the monitoring interface of the node
PLC system in real time and transmitted to the company's centralized control center through the network for storage and displayed on the monitoring main interface in real time. In the other way, the centralized control center directly retrieves the data from the upper computer database of the node PLC monitoring subsystem and displays it on the monitoring interface in real time. This data storage method of the system not only provides a data backup method, but also provides a means to verify the validity of the data. For example, when an accident occurs during the operation of a node's boiler, the data stored in the PLC host of the node can be compared with the data stored in the centralized control center to prevent data tampering and help determine the responsibility for the accident. In the specific design, this article uses the first data storage method.

In addition to the real-time upload of boiler operating parameters to the host computer system during the operation of the monitoring system, the control commands of the monitoring center to the nodes are also transmitted to the on-site node PLC controller in real time. When the upper computer and the lower computer are transmitting data, they complete the whole work according to the software flow in Figure 4.

4.4. Host Computer Network Release. The main function of network publishing is to realize the network browsing of the Kingview project, that is, to realize Internet/Intranet access. You configure the WEB publishing project in the configuration software and first configure the network for the data server in the project browser.

You select the "Networking" mode in the network parameter property page and enter the node name 219.224.14.78 corresponding to this machine in the local node. Then, you check each option when configuring the node type, because we use a Kingview project to act as all the servers required by the data server. Secondly, we set up the WEB publishing screen project and then configure the WEB server, including network parameters, node types, and client configuration. On the Kingview server, users can publish real-time information, historical information, and database information in real time. On the browser client, users can view real-time information, historical information, database information, and user management information by logging in to the main WEB interface.

In this article, the system's network publishing is through the use of a fixed IP (219.248.11.73) for WEB public network publishing. Therefore, you must go to the telecommunications department to apply for opening port 80 . Then, you connect the public network directly to the data server (the data server and the WEB server are on the same server), and you can start network publishing. According to PLC's actual demand for on-site data collection of the 19 subnodes of the hot water boiler, the information of each collection point is uploaded to the designated server IP. You store the collected information in the corresponding IP database server, use the configuration software client to load, and then use the Kingview Web publishing function to publish the relevant screens, reports, and databases on the network. 


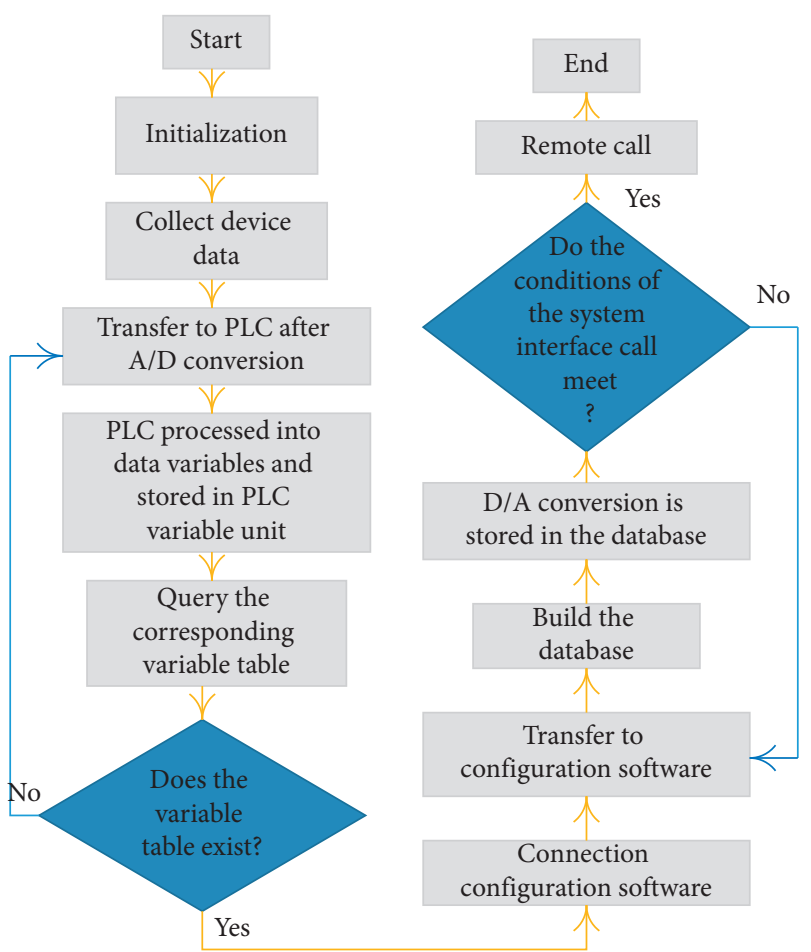

FIGURE 4: Storage of boiler operating status data.

Once the amount of data is relatively large, we can also store real-time images, daily reports, and real-time curves on different servers in the centralized control center. Each server has a fixed IP address. These servers are networked through a local area network to form the main server. The remote monitoring center can view the content you want to view by accessing different IP addresses. At the same time, we can also browse the project text of the hot water boiler that we have built in the IE browser client by adding trusted sites.

\section{Control System Parameter Tuning and Performance Simulation}

5.1. Control System Parameter Tuning. If we want to evaluate the control quality of a regulation system, we must observe its transition process under the same step disturbance. When composing the control system, we must determine the static and dynamic characteristics of each channel of the object and, at the same time, determine the control plan. Therefore, the control quality mainly depends on the tuning of the controller parameters. The task of parameter tuning is to appropriately adjust the proportional coefficient KP, integral coefficient KI, and differential coefficient KD (or proportional gain $\mathrm{KC}$, integral time TI, and derivative time TD) of the controller according to a certain control loop scheme to obtain a quality production process. Generally speaking, we always hope that the system adjustment process can be "stable, accurate, and fast." But according to automatic control theory, it is difficult to meet, so it can only be based on the quality requirements of a specific production process.
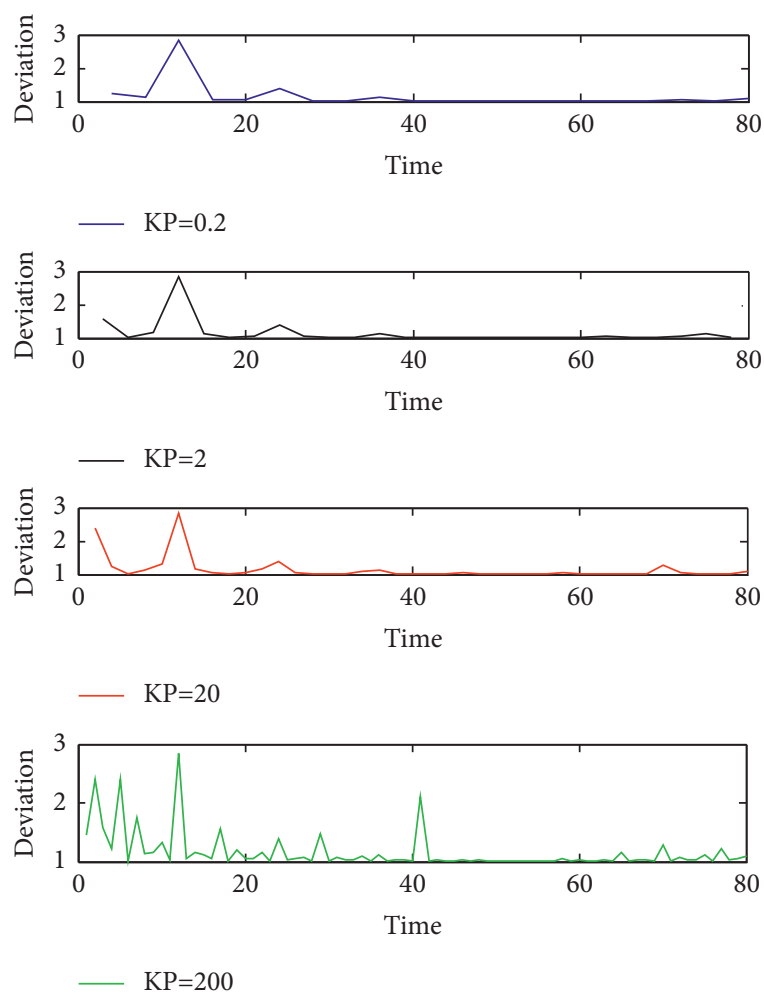

FIgURE 5: The influence of $\mathrm{KP}$ on the transition process $(\mathrm{KI}=\mathrm{KD}=0)$.

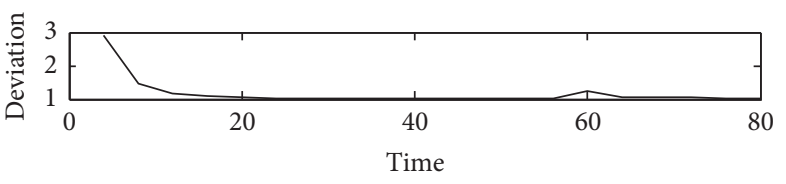

$\mathrm{KI}=0.5$
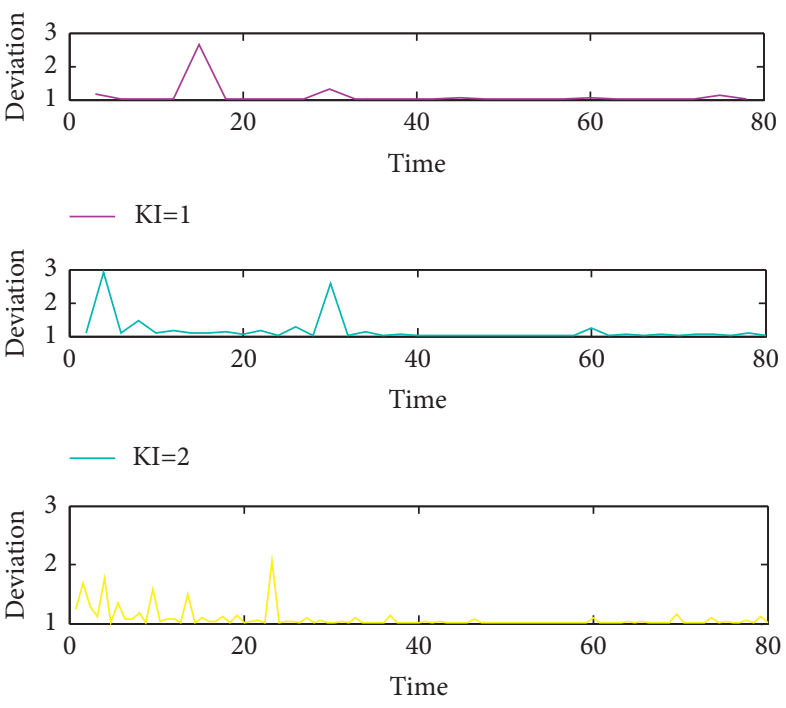

$\mathrm{KI}=4$

FIgURE 6: The impact of $\mathrm{KI}$ on the transition process $(\mathrm{KP}=2$, $\mathrm{KD}=0)$. 
The idea of the engineering tuning method is "look at the curve, adjust the parameters," which is based on the experiment, record the transition process curve and obtain the data, then look up the table to obtain the PID parameters of the regulator, and finally "look at the curve" in the system to obtain the "best" transition process that meets the process quality requirements. Because engineering tuning is an experimental trial and error method, in order to quickly make a trial, you must master the law of the controller's proportional coefficient $\mathrm{KP}$, integral coefficient KI, and differential coefficient $\mathrm{KD}$ to the transition process curve to reduce the blindness of trial and error.

Figure 5 shows that as KP changes from small to large, the dynamic deviation of the system becomes smaller, the stability becomes worse, and the system duty cycle becomes smaller. Figure 6 shows that as KI changes from small to large, the integral action increases, and the transition process of the system changes from stable to oscillation, and the overshoot increases. The integral action can eliminate the deviation, which is the most important feature of the integral action. Figure 7 shows that, with the increase of $\mathrm{KD}$, the differential effect increases, the overshoot of the closed-loop system decreases, and the system response speed becomes slower.

In addition, before the control system is put into operation, in addition to the preparation of the control system equipment itself, the production and operating conditions of the controlled boiler, the operating status of the process equipment, and the actuators directly related to the control function should also be grasped. Therefore, it is necessary to conduct field experiments within the scope of the boiler production process permit to obtain the characteristic parameters of each control object and provide a basis for parameter setting during the commissioning process.

\subsection{Simulation and Parameter Tuning of Water Supply} Control System. At $T=0 \mathrm{~s}$, a step disturbance of $-2.2 \mathrm{t} / \mathrm{h}$ of steam flow is added, and a step disturbance of $-2.2 \mathrm{t} / \mathrm{h}$ of feedwater flow is added at $T=300 \mathrm{~s}$. In the PID parameter tuning module of IBCCS-E, multiple parameter settings and comparisons can be performed on the same controller until the result is satisfied. The principle of tuning is to tune the inner loop first, and then the outer loop. Here, the tuning result of the inner loop is directly given. We select the PID1 of the feedwater cascade main controller, its PID parameters before tuning are $\mathrm{KP}=3, \mathrm{KI}=0.1, \mathrm{KD}=0$, and set its new PID parameters as $\mathrm{KP}=15, \mathrm{KI}=0.2, \mathrm{KD}=0$. The system will give the response curve of the drum water level and feedwater flow before and after the setting, as shown in Figure 8.

It can be seen from Figure 8 that after a step disturbance of $-2.2 \mathrm{t} / \mathrm{h}$ occurs in the steam flow at $T=0 \mathrm{~s}$, the feedwater flow tends to $2 \sim 6 \mathrm{t} / \mathrm{h}$ after an oscillation process; the drum water level still recovers after an oscillation process. Compared with the status before and after adjustment, the adjustment time of the system after adjustment is significantly shorter, the adjustment speed is accelerated, the overshoot of the drum water level is significantly reduced, the overshoot of the feedwater flow is not much different from that before the setting, and the adjustment process is greatly improved.
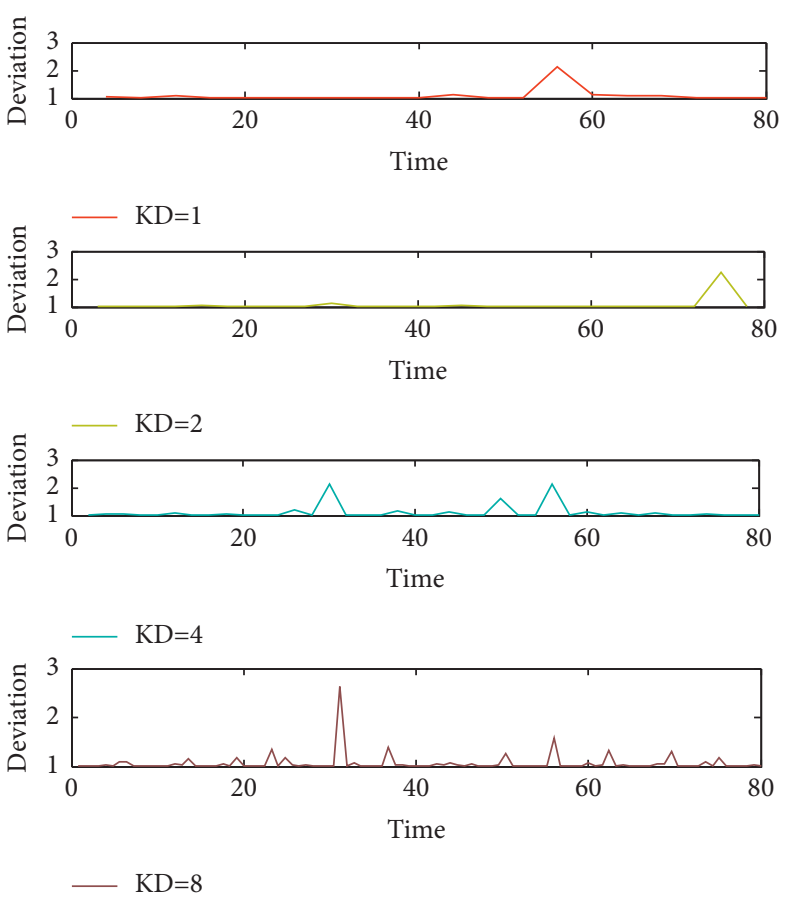

FIgURE 7: The influence of $\mathrm{KD}$ on the transition process $(\mathrm{KP}=2$, $\mathrm{KI}=0.5$ ).

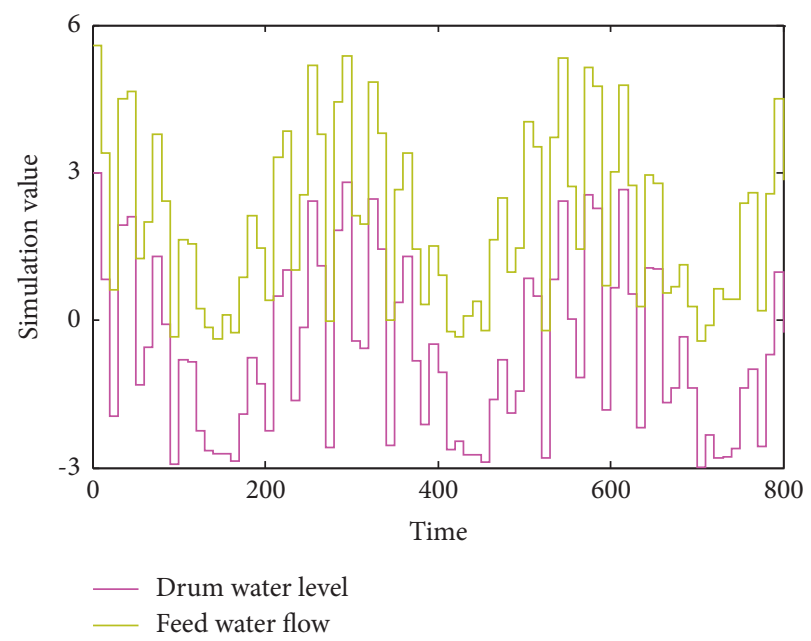

Figure 8: Simulation results of the main controller of the water supply control system.

After a step disturbance of $-2.2 \mathrm{t} / \mathrm{h}$ occurs in the feedwater flow at $T=300 \mathrm{~s}$, the secondary circuit acts as a followup system, and its given value is the output of PID1 of the main controller. Before parameter setting, the output of PID1 cannot be stabilized immediately However, the feedwater flow rate still fluctuates slightly for a long time; after the parameters are set, the output of PID1 stabilizes immediately, and finally, the feedwater flow rate and drum water level hardly move, and the feedwater flow disturbance is quickly eliminated.

It can be seen that the control quality after tuning is significantly better than the control quality before parameter 


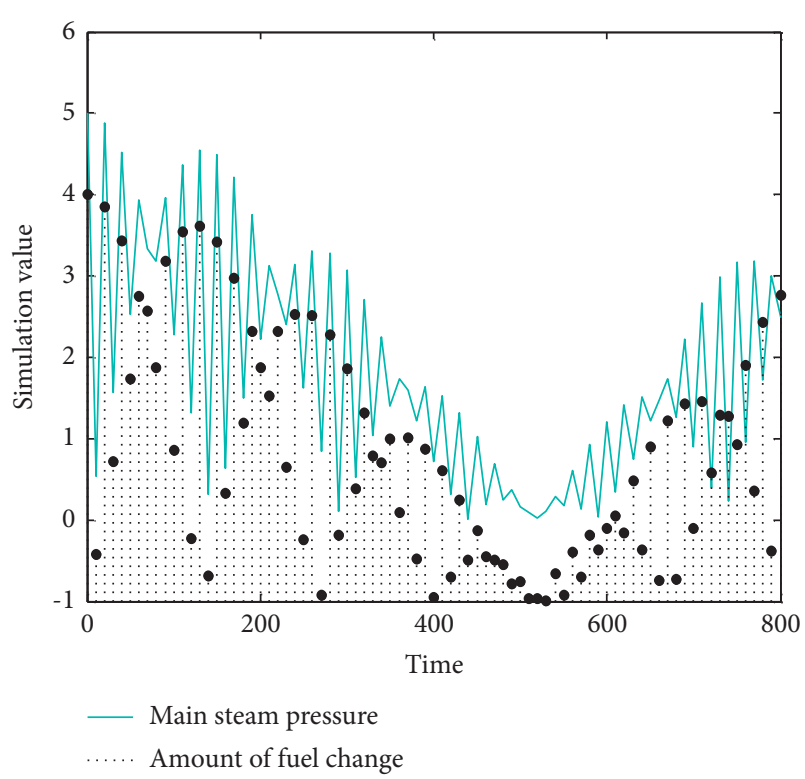

Figure 9: Parameter tuning and simulation results of the main steam pressure controller of the combustion control system.

tuning. Therefore, the parameters of PID1 are finally determined as $\mathrm{KP}=2, \mathrm{KI}=0.2$, and $\mathrm{KD}=0$.

\subsection{Simulation and Parameter Tuning of Combustion Control} System. At $T=0 \mathrm{~s}$, a step disturbance of $30 \mathrm{~kg} / \mathrm{h}$ of fuel quantity is added, and a step disturbance of steam load of $-1 \mathrm{t} / \mathrm{h}$ is added at $T=530 \mathrm{~s}$. In the PID parameter tuning module of IBCCS-E, multiple parameter settings and comparisons can be performed on the same controller until the result is satisfied. The setting sequence is to set the induced air regulation subsystem first, then the air supply regulation subsystem, then the fuel quantity control subsystem, and finally the main steam pressure control loop. We select $\mathrm{PID} 3$, its parameters before tuning are $\mathrm{KP}=20$, $\mathrm{KI}=0.5, \mathrm{KD}=0$, and set its new parameters as $\mathrm{KP}=5$, $\mathrm{KI}=0.1, \mathrm{KD}=0$. Figure 9 shows the response curve of main steam pressure and fuel quantity.

It can be seen from Figure 9 that after a step disturbance occurs in the fuel quantity at $T=0 \mathrm{~s}$, the fuel quantity feedback signal will change, causing the signal imbalance at the inlet of the fuel regulator PID4 to change its output signal and actuate the fuel actuator. The fuel quantity is quickly restored to the value before the disturbance. Before and after tuning, although the main steam pressure overshoot is small before tuning, the main steam pressure and fuel volume will both produce continuous small oscillations; the overshoot of the system after tuning is not much different from that before tuning, and the adjustment trend slows down. No oscillations will occur, and the adjustment process will be improved.

After a step disturbance of $-1 \mathrm{t} / \mathrm{h}$ occurs in the steam flow at $T=900 \mathrm{~s}$, the main steam pressure Pm changes immediately and deviates from the set value, and after another oscillation process, it eventually returns to the set value; the fuel quantity goes through an oscillation process.
After that, it finally stabilizes at a new stable value that is compatible with the steam flow rate. Compared with before and after tuning, although the main steam pressure overshoot is small before tuning, the main steam pressure and fuel quantity will both produce continuous small amplitude oscillations; the overshoot of the system after tuning is slightly larger than before tuning, and the adjustment trend slows down. But in the end, it reaches a stable state, no oscillation occurs, and the adjustment process is improved.

\section{Conclusion}

This paper designs the main monitoring screens, daily reports, real-time trend graphs of main parameters, alarm windows, and other screens of industrial hot water boilers and realizes the remote monitoring of hot water boiler data and real-time release of images using the network release provided by KingView. The focus is on the data collection work of the hot water boiler monitoring system and the design of the upper computer man-machine interface. This article introduces the method of parameter tuning of the control system, establishes a simulation model of the industrial boiler computer control system, provides an interface between the host computer control software and the simulation model, conducts a controller parameter tuning simulation experiment, and carries out the experimental results analysis. The upper computer monitors the status of the two lower computers in real time and provides the fault information of the lower computers in time. Once the faulty lower-level computer is repaired, it will automatically start to the standby state once it is powered on. This structure decentralizes the functions of the system and improves reliability. The system can also flexibly increase or decrease input and output modules to realize one machine one control (one lower machine controls one boiler) or one machine multicontrol (one lower machine controls multiple boilers). The other parts have no effect. The system configuration is flexible, and the expandability is good. Because the collection signal type of the hot water boiler monitoring system is relatively simple, it is limited to the signal collection related to the control element, such as the collection of the input and output point status of the PLC, the collection of the status of the internal auxiliary relay, and the collection of the data in the data register. However, some parameters such as self-starting and manual, PID control constant temperature and pressure are not considered in the design. If you can study the PID control and participate in the control, it will be a new breakthrough in the future.

\section{Data Availability}

The data used to support the findings of this study are available from the corresponding author upon request.

\section{Conflicts of Interest}

There are no conflicts of interest in this article. 


\section{Acknowledgments}

The study was supported by Shijiazhuang University Development and Construction Projects of Applied Coursework (YYKC-202003); Shijiazhuang University Teaching Reform Research and Practical Projects (JGXM-202155G); and Hebei Province Applied Technology University Seminar Project (JY2021023).

\section{References}

[1] Y. Jiang, S. Yin, and O. Kaynak, "Data-driven monitoring and safety control of industrial cyber-physical systems: basics and beyond," IEEE Access, vol. 6, pp. 47374-47384, 2018.

[2] H. M. La, T. H. Dinh, N. H. Pham, Q. P. Ha, and A. Q. Pham, "Automated robotic monitoring and inspection of steel structures and bridges," Robotica, vol. 37, no. 5, pp. 947-967, 2019.

[3] Y. Cao, L. Ma, and Y. Zhang, "Application of fuzzy predictive control technology in automatic train operation," Cluster Computing, vol. 22, no. 6, pp. 14135-14144, 2019.

[4] V. Tsakanikas and T. Dagiuklas, "Video surveillance systemscurrent status and future trends," Computers \& Electrical Engineering, vol. 70, pp. 736-753, 2018.

[5] M. Ali, S. Qaisar, M. Naeem, and S. Mumtaz, "Energy efficient resource allocation in D2D-assisted heterogeneous networks with relays," IEEE Access, vol. 4, pp. 4902-4911, 2016.

[6] C. P. Janssen, S. F. Donker, D. P. Brumby, and A. L. Kun, "History and future of human-automation interaction," International Journal of Human-Computer Studies, vol. 131, pp. 99-107, 2019.

[7] O. Carsten and M. H. Martens, "How can humans understand their automated cars? HMI principles, problems and solutions," Cognition, Technology \& Work, vol. 21, no. 1, pp. 3-20, 2019.

[8] B. Li, R. Lu, W. Wang, and K.-K. R. Choo, "Distributed hostbased collaborative detection for false data injection attacks in smart grid cyber-physical system," Journal of Parallel and Distributed Computing, vol. 103, pp. 32-41, 2017.

[9] H. C. Lee and K. H. Ke, "Monitoring of large-area IoT sensors using a LoRa wireless mesh network system: design and evaluation," IEEE Transactions on Instrumentation and Measurement, vol. 67, no. 9, pp. 2177-2187, 2018.

[10] B. F. Spencer Jr., V. Hoskere, and Y. Narazaki, "Advances in computer vision-based civil infrastructure inspection and monitoring," Engineering, vol. 5, no. 2, pp. 199-222, 2019.

[11] M. Hossain, S. M. R. Islam, F. Ali, K.-S. Kwak, and R. Hasan, "An internet of things-based health prescription assistant and its security system design," Future Generation Computer Systems, vol. 82, pp. 422-439, 2018.

[12] R. Casado-Vara, P. Chamoso, F. De la Prieta, J. Prieto, and J. M. Corchado, "Non-linear adaptive closed-loop control system for improved efficiency in IoT-blockchain management," Information Fusion, vol. 49, pp. 227-239, 2019.

[13] A. Mosenia, S. Sur-Kolay, A. Raghunathan, and N. K. Jha, "Wearable medical sensor-based system design: a survey," IEEE Transactions on Multi-Scale Computing Systems, vol. 3, no. 2, pp. 124-138, 2017.

[14] R. Y. Zhong, L. Wang, and X. Xu, "An IoT-enabled real-time machine status monitoring approach for cloud manufacturing," Procedia CIRP, vol. 63, pp. 709-714, 2017.

[15] G. Nagarajan and R. I. Minu, "Wireless soil monitoring sensor for sprinkler irrigation automation system," Wireless Personal Communications, vol. 98, no. 2, pp. 1835-1851, 2018.
[16] D. Zhang, "High-speed train control system big data analysis based on the fuzzy RDF model and uncertain reasoning," International Journal of Computers Communications \& Control, vol. 12, no. 4, pp. 577-591, 2017.

[17] M. Heimberger, J. Horgan, C. Hughes, J. McDonald, and S. Yogamani, "Computer vision in automated parking systems: design, implementation and challenges," Image and Vision Computing, vol. 68, pp. 88-101, 2017.

[18] I. Y. Noy, D. Shinar, and W. J. Horrey, "Automated driving: safety blind spots," Safety Science, vol. 102, pp. 68-78, 2018.

[19] A. F. Hussein, N. A. Kumar, M. Burbano-Fernandez, G. RamirezGonzalez, E. Abdulhay, and V. H. C. De Albuquerque, "An automated remote cloud-based heart rate variability monitoring system," IEEE Access, vol. 6, pp. 77055-77064, 2018.

[20] S. Rodríguez, T. Gualotuña, and C. Grilo, "A system for the monitoring and predicting of data in precision agriculture in a rose greenhouse based on wireless sensor networks," Procedia Computer Science, vol. 121, pp. 306-313, 2017.

[21] M. Riahi Manesh and N. Kaabouch, "Analysis of vulnerabilities, attacks, countermeasures and overall risk of the automatic dependent surveillance-broadcast (ADS-B) system," International Journal of Critical Infrastructure Protection, vol. 19, pp. 16-31, 2017.

[22] M. Shahid, L. Henrik, M. S. H. Kazi, R. Jonathan, and R. Ayman, "Smart direct-LTE communication: an energy saving perspective," Ad Hoc Networks, vol. 12, pp. 296-311, 2014.

[23] M. Z. Khan, S. Harous, S. U. Hassan, M. U. Ghani Khan, R. Iqbal, and S. Mumtaz, "Deep unified model for face recognition based on convolution neural network and edge computing," IEEE Access, vol. 7, pp. 72622-72633, 2019. 